\title{
An Integrated Model in E-Government Based on Semantic Web, Web Service and Intelligent Agent
}

\author{
Hongtao $\mathrm{Zhu}^{1}$, Fangli Su${ }^{2}$ \\ 1 Department of Information Science, Zhengzhou Institute of \\ Aeronautical Industry Management \\ Zhengzhou, China, Postcode: 450015 \\ pds_zhht@126.com \\ 2 Department of Information Science, Zhengzhou Institute of \\ Aeronautical Industry Management \\ Zhengzhou, China, Postcode: 450015 \\ suli_2000@163.com
}

\begin{abstract}
One urgent problem in E-government service is to improve service efficiency through breaking information islands while constructing integrated service systems. Web Service provides a set of standards for the provision of functionality over the Web, and Web Service descriptions are pure syntactic instead of semantic content. Semantic Web provides interoperability from syntactic level to semantic one not only for human users but also for software agents. Semantic Web and Intelligent Agent are highly complementary, and the existing technologies have made their unification quite feasible, which brings about a good opportunity to the development of E-government. Based on Semantic Web and Intelligent Agent technologies an integrated service model of E-government is suggested in this paper.
\end{abstract}

\section{Introduction}

E-Government applications often require affording united information through integrating resources from all kinds of sources, or combining a few component software systems from the same or different institutions and organizations into one distributed software system. To the end user, the whole system should be felt like one single service system [1]. Nowadays most E-Government services are usually offered directly by different government organizations, or by the-third-partnerinformation service provider (ISP). Because of relying on different basic technologies, they do not have an interface for software system integration. This

Please use the following format when citing this chapter.

Thu. II., Su, F., 2007, in IFIP International lederation for Information Processing, Volume 252, Integration and Innovation Orient to E-Society Volume 2, eds. Wang, W., (Boston: Springer), pp. 96-102. 
makes it difficult to communicate, to share and to integrate information for the institutions, and also the end user. On the other hand, most of E-government information on the internet can't be understood and processed by computer automatically but only by human beings. The degree of automation and intelligence is rather low, which slows the velocity of information circulation and results in low efficiency of E-government services. Sufficient E-government applications should be an architecture that can be used to automate the routine petition process which includes analyses of users and their queries, information retrieval, integration of searching results [2], and so on. In this article the authors suggest an integrated service model of E-government based on Semantic Web and Intelligent Agent technologies, which is indispensable during constructing sharing systems of Egovernment.

There are four sections in this paper. In section 1, the present condition of Egovernment is introduced briefly. In section 2, the feasibility of combination of Semantic Web, Web Service and Intelligent Agent is discussed while explaining their features and functions. In section 3, the authors propose an integrated model of E-government services and illustrate its framework. In section 4, a conclusion is given.

\section{Technologies related}

\subsection{Semantic Web}

According to the World Wide Web Consortium (W3C), who defines the Semantic Web as "The Semantic Web provides a common framework that allows data to be shared and reused across application, enterprise, and community boundaries" [3], the Semantic Web is about two things. First, it is about common formats for integration and combination of data drawn from diverse sources, where on the original Web mainly concentrated on the interchange of documents. Second, it is about a language for recording how the data relates to real world objects. That allows a person, or a machine, to start off in one database, and then move through an unending set of databases which are connected not by wires but by being about the same thing. Its frame is like a layered cake [4] (See Fig.1).

At present the above three layers (logic, Proof, and Trust) in Fig.1 are still under discussion, the other four layers are widely used in many fields, even if some key technologies are rather less stable.

It is obvious that Semantic Web allows computers to track links and facilitate the integration of information from many different sources. So the Semantic Web initiative aims to resolve sharing problems from the information perspective. There exist many problems in present E-government Service system that could be settled by Semantic Web. 


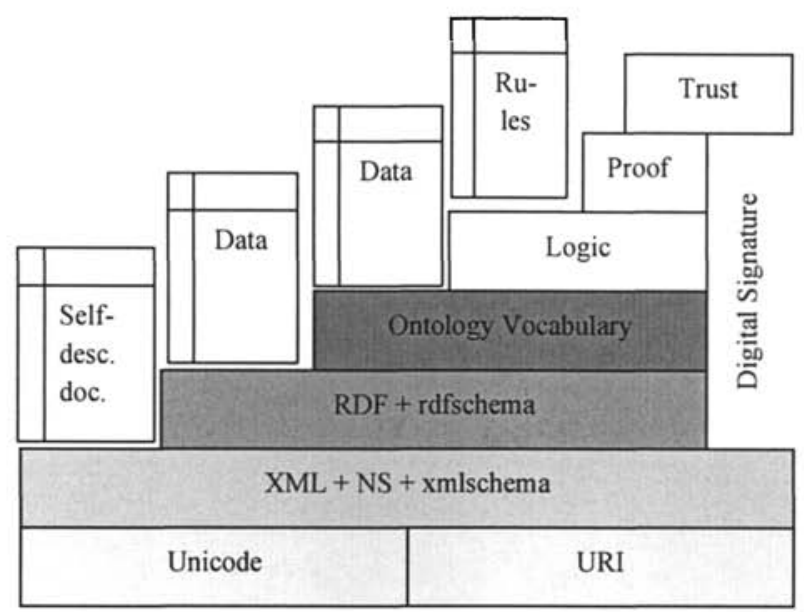

Fig. 1. The frame of Semantic Web

\subsection{Supplementary of Semantic Web and Web Service}

Web Service provides a set of standards for the provision of functionality over the Web: the specification of the SOAP (Simple Object Access Protocol) as a standard for transmitting messages, the WSDL (Web Services Description Language) as a standard for describing interfaces provides platform-independent access to back-end functionality, and the UDDI (Universal Description, Discovery and Integration) as a standard for describing, promulgating and integrating services. These standards are the core of Web Service. Together, the Web infrastructure and Web Service descriptions have the functions of reducing the cost and time of integrating applications and integrating distributed information, because with the Web Service there is no need to build customized communication lines, to implement proprietary messaging protocols, and to interpret the information from different government respective department [5].

However, Web Service descriptions are usually syntactic instead of semantic content, which leads to necessity of much man-intervention when to decide whether it offers the desired functionality. Furthermore, if E-government services have different interaction styles or use different terms for the description of the data formats and functionality, there will be no way of cooperating.

The improvement of efficiency in the use of Web Service can be attained if it is combined with Semantic Web technology. The common goal of Semantic Web and Web Service is to create automatic and intelligent services and E-government processing infrastructure by using web contents which can be understood by both people and computers. Therefore, the realization of function supplementary is one 
kind of natural choice, with which service providers and end users enable dynamically to locate partners they want, and to promote cooperation with them.

\subsection{Integration of Intelligent Agent and Semantic Web}

Nowadays, although there has not been a single universally accepted definition, the term "Agent" is widely used by many experts working in related areas, especially in the fields of computer science and artificial intelligence (AI). In computer science, an Intelligent Agent (IA) is generally a software agent that assists users and will act on their behalf, in performing non-repetitive computer-related tasks [6]. It has multitudinous merits, such as independency, openness, and so on. It is usually used in the system which is open, distributed, and logic. Just as what Michael Wooldridge and Nick Jennings wrote in their article [7], now we can feel that Intelligent Agent has been a key technology as computing systems become ever more distributed, interconnected, and open. In current web environments, the ability of agents to autonomously understand, to cooperate, coordinate, and negotiate with others, and to respond flexibly and intelligently to dynamic and unpredictable situations will lead to more convenience for users. While Intelligent Agent accomplishing various tasks, it is rather important to estimate and comprehend semantic environment exactly. Semantic Web has provided favorable environment for Intelligent Agent. If end users hope to access distributed web information through Intelligent Agent, Ontology Vocabulary, one part of Semantic Web, must be used. Higher grade applications of Intelligent Agent will connect web information with related knowledge and rules, and then achieve useful information from the web. The integration of Intelligent Agent technology and ontologies Semantic Web, could significantly affect the use of web services and the ability to extend programs to perform tasks for users more efficiently and with less human intervention [8].

\section{E-government Service Integrated Model Based on Semantic Web, Web Service and Intelligent Agent}

In this section, the authors propose an E-government Service Integrated Model based on the technologies of Semantic Web, Web Service and Intelligent Agent (See Fig.2).

The model can associate all levels of government information system and provide services for end users with unified interface. From the point of theory, the model makes the semantic content of web resources (including information and services) more clear and perfect and enables computers to understand more exactly. With this model the distributed information or service with different descriptionformat can be processed more automatically and intelligently. With the aid of SOAP, UDDI and WSDL, we can unify Semantic Web and Web Service to realize loose coupling among E-government services. The colligation of Semantic Web and Intelligence Agent reduces human-intervention and facilitate the realization of automation.

It is evident that our integrated model architecture may be divided into four levels, namely: (I) E-government Resource Providers Level, which provides all kinds 
of information or services that may be described with different formats; (II) Mediation Level, which supports the communication of G2G and G2C with the aid of the DAML-S/Matchmaker [9], agent and ontologies; (III) Interaction Level, which

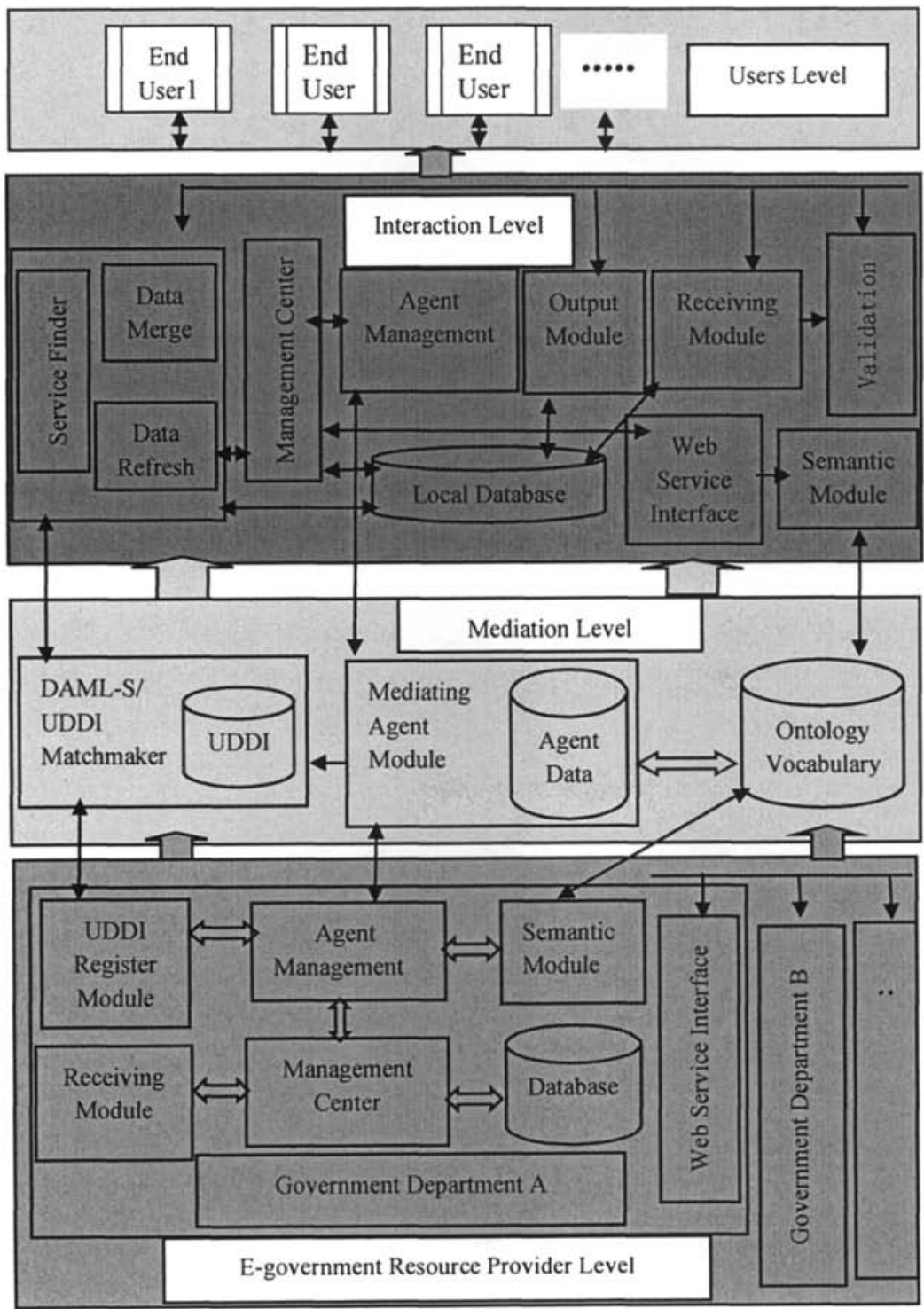

Fig.2. E-government Service Integrated Model 
provides suitable information or services for end users with the right forms. (IV) Users Level, which provides interface system. Every Level has several modules which implement some specific functions. Another point that should be noticed is that every level except the fourth one in this model has its own Intelligent Agent. There are three kinds of agents in all: intelligent user agents, E-government local information agents and mediation agents. Information that all the agents hold has specific semantic meaning so that cooperation is done well among the agents in order to achieve certain service.

In the following sub-sections we describe in detail all these components. Egovernment Resource Providers Level is the bases of the whole model, which may operate on different servers because they may not be from the same government department. There are a few modules as following. They seal their information or services they provide with UDDI Register Module, and then register in it after giving descriptions with DAML-S. Furthermore, they usually provide the catalog of government information or their services items. Receiving Module is in chare of receiving, register, modify and delete user's query record. Web Service Interface Module provides foreign interface while displaying government information or services lists. Semantic Module is used to assure semantic unification between interior system and exterior system and to afford semantic aids to other modules. Agent Management Module manages to interior agents and their information exchange with foreign agents (such as mediation query agent). Management Center harmonizes interactions of all the modules and intervenes with the process if it is necessary.

Mediation Level includes DAML-S/Matchmaker, Mediation Agent database and Ontology. DAML-S/Matchmaker is a frame of Semantic Web Service register and discovery based on DAML-S. It can implement bidirectional information match. Mediation Agent Module is responsible to harmonize end user agent and Egovernment local agent. Ontology provides sharing concept model which includes a vocabulary with semantic relations.

Interaction Level acts as a bridge that connecting $\mathrm{G} 2 \mathrm{G}$ or G2C. Validation Module is used to know user's qualification. Output Module is responsible to transfer classified information or service information to users' interface. Furthermore, the provided content can be changed dynamically according to users' need. Receiving Module collects and registers users query and then transfers them to Egovernment Providers. Web Service Interface Module integrates the catalog of Egovernment resources or service information, and then stores them in local database. Service Finder Module can seek the new provided information in UDDI, afterward, put the results into the local database. Functions of Semantic Module and Agent Management Module in this level are similar as those in E-government Service Providers Level mentioned above. The content of intercommunion in the whole system is transferred to the end users through Users Level.

Contrast to the other model related, this E-government integrated model has following distinct merits at least:

\footnotetext{
$>$ Distributional Application

$>$ Platform Irrelevant

$>$ High Integration of Different-structured Government Information

$>$ E-government One Station Service
} 
$>$ Resources and Services Sharing and Cooperation

\section{Conclusion}

Present E-government solutions often require a costly and custom hardware and software infrastructure for both department of cooperating partmers and end users. Furthermore, the lack of formal and unified descriptions of services offered by organizations hampers automation in the location and usage of services required to perform a Government information or services access activity. In this paper the authors discuss the feasibility of integration of several technologies, including Semantic Web, Web Service and Intelligent Agent, after analyzing their own features. And then a new E-government integrated model is brought forward. This model provides a framework for the integration and sharing of distributed and differentstructured E-government resources. It enables seamless E-government services integration through formal descriptions, maximal decoupling of components, and strong intelligent cooperating support. However, realization of some technical details should be further researched, which is our next work recently.

\section{References}

1. J. Korhonen, L. Pajunen and J. Puustjärvi, "Requirements for Using Agent-Based Automation in Distributed E-Government Applications", R. Traunmüller (Ed.): EGOV, LNCS 2739,157-160 (2003).

2. R. Traunmüller and M. Wimmer, Directions in E-Government: Processes, Portals, Knowledge, Proceedings of the 12th International Workshop on Database and Expert Systems Applications (IEEE Computer Society, Washington, DC, 2001), 313-317.

3. W3C, "Semantic Web" (April 25, 2007). http://www.w3.org/2001/sw.

4. R. Klischewski, "Semantic Web for E-Government", $R$. Traunmüller (Ed.): EGOV, LNCS 2739, 288-295 (2003).

5. G. Alonso, F. Casati, H. Kuno and V. Machiraju, "Web Services: Concepts, Architecture and Applications", Springer Verlag, Berlin, 123-149(2004).

6. Wikipedia, "Intelligent agent "(July 8, 2007). http://en.wikipedia.org/wiki/Intelligent_agent.

7. M. Wooldridge and N. Jennings, "Intelligent Agent: Theory and practice", Knowledge Engineering Review 10 (2), 115-152 (1995).

8. J. Hendler, "Agents and the Semantic Web", IEEE Intelligent Systems, March/April, 30-37 (2001). http://oopsla.snu.ac.kr/ jnkim/pdf/IS_2.pdf.

9. S. Katia, P. Massimo, A. Anupriya and S. Naveen, "Automated discovery, interaction and composition of Semantic Web Services", Web Semantics 1 (1), 27-46 (2003). 